\title{
An Efficiency-Aware Continuous Adaptive Proportional-Integral Velocity-Feedback Control for Wave Energy Converters
}

\author{
Hoai-Nam Nguyen ${ }^{a}$ Paolino Tona ${ }^{\mathrm{a}}$ \\ ${ }^{a}$ Control, Signal and System Department - IFP Energies nouvelles, Rond-point de \\ l'echangeur de Solaize BP3, 69360 Solaize, France
}

\begin{abstract}
The main objective in hydrodynamic control of wave energy converters (WECs) is the maximization of the energy captured from the waves. Latching control, model predictive control and "PI" control are examples of implementable strategies surveyed in the literature. "PI" control is the common name of a form of hydrodynamic control where the control force applied to the captor is a proportional-integral feedback of captor velocity. While suboptimal, it has the merit of being simple, requiring only straightforward computations and can be considered a standard solution for WECs with a four-quadrant power takeoff (PTO) system. Adaptive "PI" control has been already discussed in the literature, usually using a gain-scheduling approach, with optimal gains precomputed off-line for a representative set of sea states and applied as a function of estimated sea state conditions. In most literature, only average on-line estimations of sea states have been proposed, with time windows of several minutes. Such intermittent adaptive control laws are clearly suboptimal in terms of energy recovery, since the control gains are not continuously updated whereas the sea state is continuously time-varying. In this paper we present a continuously adaptive "PI" control strategy, whose gains are adapted on-line on a wave-to-wave basis, based on a real-time estimate of the dominant wave frequency of the wave force. The PTO efficiency is taken into account. The proposed control method is validated and compared through experiment for irregular sea states.
\end{abstract}

Key words: Wave Energy Converters, Adaptive Control, PTO Efficiency, Wave Force Estimation, Wave Dominant Frequency

Email addresses: hoai-nam.nguyen@ifp.fr (Hoai-Nam Nguyen), paolino.tona@ifp.fr (Paolino Tona). 


\section{Introduction}

A wave energy converter (WEC) is a device used to produce electrical energy from wave-induced motion. Several studies have shown that one of the key aspects for maximizing the energy yield of many WECs is the control of the 5 dynamic response of the device to wave conditions, also defined as hydrodynamic control [17]. More precisely, we consider here the response control of the WEC captor, whose task is to transfer energy from wave to oscillating body, via the power-take off (PTO) system, responsible of a further energy conversion, generally to electricity. A schematic example of a WEC of the 10 point absorber type is shown in Figure 1: a float (the captor) oscillates under the action of waves and is connected to a PTO system; the PTO, by exercising an appropriate force on the float, converts its mechanical energy into electrical energy. The PTO can be a simple linear electric generator, or a more complex multi-stage device, such as, for instance, a hydraulic motor connected to a rotary electric generator. Hydrodynamic control of such a WEC can be performed using the PTO as an actuator and adjusting the force it exercises on the oscillating body serving as a captor, in order to maximize the extracted energy.

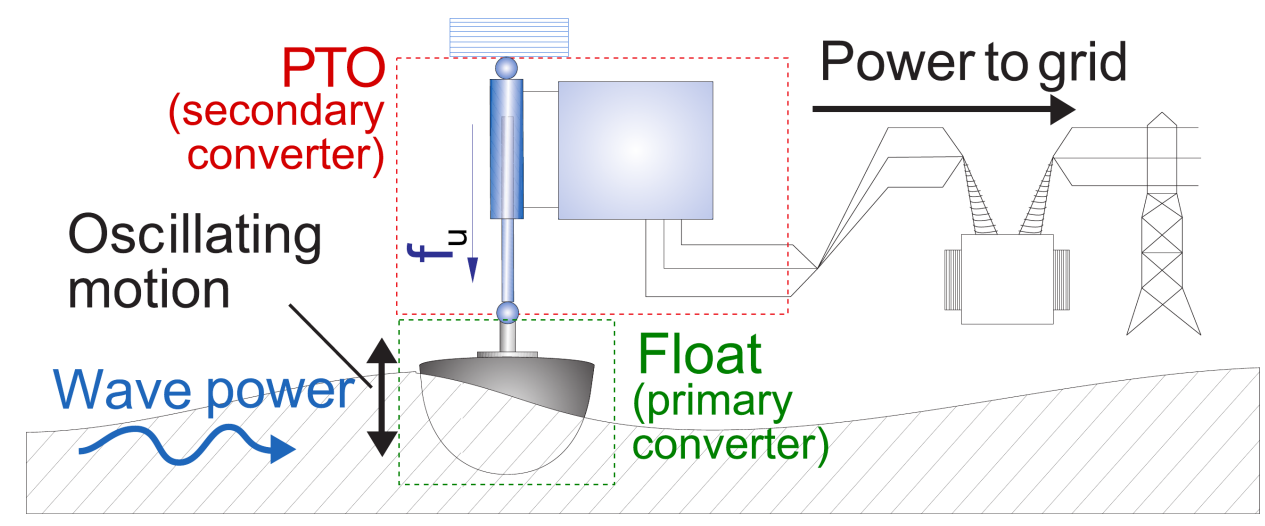

Fig. 1. Schematic diagram of a generic (heaving-buoy) wave energy converter

Among the implementable strategies surveyed in the literature, we can recall for instance, latching control, model predictive control and "PI" control. While, in theory, latching control [1] and model predictive control (MPC) [4], [19] allow to achieve high levels of energy capture, their practical implementation may be very challenging. This is due to the fact that these control strategies require a short-term prediction of wave excitation force, which can degrade the control performance, if the prediction is not perfect. Moreover, if the conversion efficiency of the PTO system is less than $100 \%$, the MPCor MPC-like optimization problem is generally nonlinear and non-convex [22], [2]. Hence the computational burden might be prohibitive for on-line implementation. On the other hand, "PI" control, where the PTO force is computed from a proportional-integral action on captor velocity, has the merit of being 
simple, requiring only straightforward computations [11]. Because of that, it was one of the first control strategy to be implemented on WEC prototypes, for instance the "Salter's duck" [25]. Even nowadays, it can be considered a standard solution, when a four-quadrant PTO, capable of both harvesting and drawing power from the grid, is available. The "P" component converts the wave energy into useful energy, while the "I" component modifies the WEC system natural frequency which allows the absorber to be more often in phase with the incoming waves, at the expense of some power being drawn from the grid. Note that the damping or "P" controller, where the PTO force is specified to be proportional to and oppositely directed to the velocity of the primary converter, is even more widely adopted, as it does not require the use of reactive power.

Assuming 100\% PTO conversion efficiency, tools involved in the computation of the optimal PI gains are greatly simplified. For the case of regular waves, 45 there exists an elegant analytical expression for the PI gains as a function of wave frequency [6]. One of the well known results is that, in order to harvest the maximum amount of energy, the PI gains should be chosen in such a way that the optimal velocity is in phase with the wave excitation force [6]. Unfortunately, a perfect PTO with no losses is unrealistic. The power that ${ }_{50}$ is withdrawn from the grid is always more expensive than the power that is delivered to the grid. In this case, the PI gains are computed off-line using a griding approach: for each sea state, the closed loop is simulated for a grid of gains, and the combination leading to the best average power is picked [26]. Clearly, this is a brute-force search, and therefore no optimality is guaranteed.

Let us underline that whatever method is used to compute the PI gains, they should not be kept constant, as the sea conditions change. Adaptive PI control has been already discussed in the literature [27], [14], [11], at different level of details, in the form of a gain-scheduling approach. The main idea is to compute "optimal" gains for a representative set of sea states. Then the appropriate control action is found from a look-up table whose input is the current sea state, which can be identified by the characteristics of its spectrum. In most literature, only average on-line estimations of sea states have been proposed, with time windows of several minutes, e.g., 10 minutes in [14], $20-30$ minutes in [11]. Such intermittent adaptive control laws are clearly suboptimal in terms of energy recovery, since the control gains are not continuously updated whereas the sea state continuously changes.

In this paper we present a new adaptive "PI" control algorithm for WECs, for which the "PI" gains are continuously adapted on-line, on a wave-to-wave basis, based on a real-time sinusoidal approximation of the wave excitation force. It has three main notable features.

(1) It is shown that the well-known result [6] stating that the optimal velocity 
should be in phase with the wave excitation force is generally not true in the case of a non-ideal PTO.

(2) This is the first time, to the best of the authors' knowledge, that an analytical expression is given for the average harvested power taking into account the PTO efficiency.

(3) It is shown how to convert the problem of computing the PI gains into a convex optimization problem. This is a huge advantage compared to solving a non-convex optimization problem.

This paper presents a rigorous treatment of the preliminary results in [23].

The paper is organized as follows. Section 2 describes the WEC modeling and defines the control problem, along with the performance criterion to be optimized and the reference intermittent adaptive "PI" control. Section 3 presents the procedure to compute the optimal frequency response of the control block under regular waves in the presence of a non-ideal PTO. Section 4 explains how the results of Section 3 can be used to deal with realistic polychromatic sea states. In Section 5, the experimental setup is described used to validate the algorithm, and the results are reported. Finally, some conclusions are drawn in Section 6.

\section{WEC Modeling and Problem formulation}

\subsection{WEC Modeling}

We consider here the type of WECs schematically represented in Fig. 1, that is, point-absorber WECs with an oscillating part that moves in one degree of freedom, the heaving direction for instance, with respect to a reference (fixed anchor or a submerged body). From the relative motion, useful energy can be extracted via the PTO.

Under the assumption that the oscillations of the system are relatively small, the WEC motion can be expressed in the frequency domain as in [6]

$$
\left(j \omega M+Z_{p a}(j \omega)+\frac{K_{p a}}{j \omega}\right) v(j \omega)=f_{e x}(j \omega)-f_{u}(j \omega)
$$

where

- $v(j \omega)$ is the heaving velocity of the oscillating part.

- $f_{e x}(j \omega)$ and $f_{u}(j \omega)$ are the wave excitation force and the PTO force, respectively.

- $M$ is the mass of the float with its connected parts. 
- $Z_{p a}(j \omega)$ is the radiation impedance.

- $K_{p a}$ is the stiffness coefficient.

Equation (1) is based on the Cummins integro-differential equation [5], whose hydrodynamic coefficients can be computed via boundary element method software, such as WAMIT, Diodore, AQWA ou NEMOH. $Z_{p a}(j \omega)$ is the result of an approximation of the radiation impulse response by an IIR filter and can be decomposed as

$$
Z_{p a}(j \omega)=B_{p a}(\omega)+j \omega\left(M_{p a}(\omega)+M_{\infty}\right)
$$

where $B_{p a}(\omega)$ is the radiation resistance, and $M_{p a}(\omega)$ is the added mass after $M_{\infty}$, the asymptotic value of the added mass for $\omega \rightarrow \infty$ is removed. As in most modeling studies on point absorbers, it is assumed that viscous, frictional forces are negligible compared to the other terms in the equation of motion. Rewrite (1) as

$$
v(j \omega)=\frac{1}{Z_{i}(j \omega)}\left(f_{e x}(j \omega)-f_{u}(j \omega)\right)
$$

where the intrinsic impedance $Z_{i}(j \omega)$ is defined as

$$
\begin{aligned}
Z_{i}(j \omega) & =B_{p a}(\omega)+j \omega\left(M+M_{\infty}+M_{p a}(\omega)-\frac{K_{p a}}{\omega^{2}}\right) \\
& =R_{i}(\omega)+j X_{i}(\omega)
\end{aligned}
$$

where

$$
\left\{\begin{array}{l}
R_{i}(\omega)=B_{p a}(\omega), \\
X_{i}(\omega)=\omega\left(M+M_{\infty}+M_{p a}(\omega)-\frac{K_{p a}}{\omega^{2}}\right)
\end{array}\right.
$$

\subsection{Control Objective}

The control objective is to maximize the average electric power produced by the WEC

$$
P_{a}=\frac{1}{T} \int_{t=0}^{T} \eta f_{u}(t) v(t) d t
$$

where $\eta$ is the efficiency coefficient.

In the literature, it is generally assumed that $\eta=1$, i.e. the PTO system is perfect [7], [12]. Unfortunately, this assumption is unrealistic. As written in the Introduction, the power that is withdrawn from the grid and used to accelerate the float is always more expensive than the power that is produced by the float motion and delivered to the grid via the PTO system. To take into account this fact, we consider the efficiency coefficient $\eta$ as a function of 
the ideal instantaneous power $f_{u} v$

$$
\eta\left(f_{u} v\right)=\left\{\begin{array}{l}
\eta_{p} \text { if } f_{u} v \geq 0 \\
\eta_{n} \text { if } f_{u} v<0
\end{array}\right.
$$

where the coefficients $0<\eta_{p} \leq 1$ and $\eta_{n} \geq 1$ depend on the PTO system, and may even be a function of $f_{u} v$. Figure 2 shows $\eta$ as a function of $f_{u} v$ for the WEC system in [26], for which $\eta_{p}=0.7, \eta_{n}=1.43$, and hence $\eta_{n} \approx 2 \eta_{p}$. This implies that it is two times more expensive to withdraw energy from the grid than to deliver energy to the grid, for the WEC prototype [26].

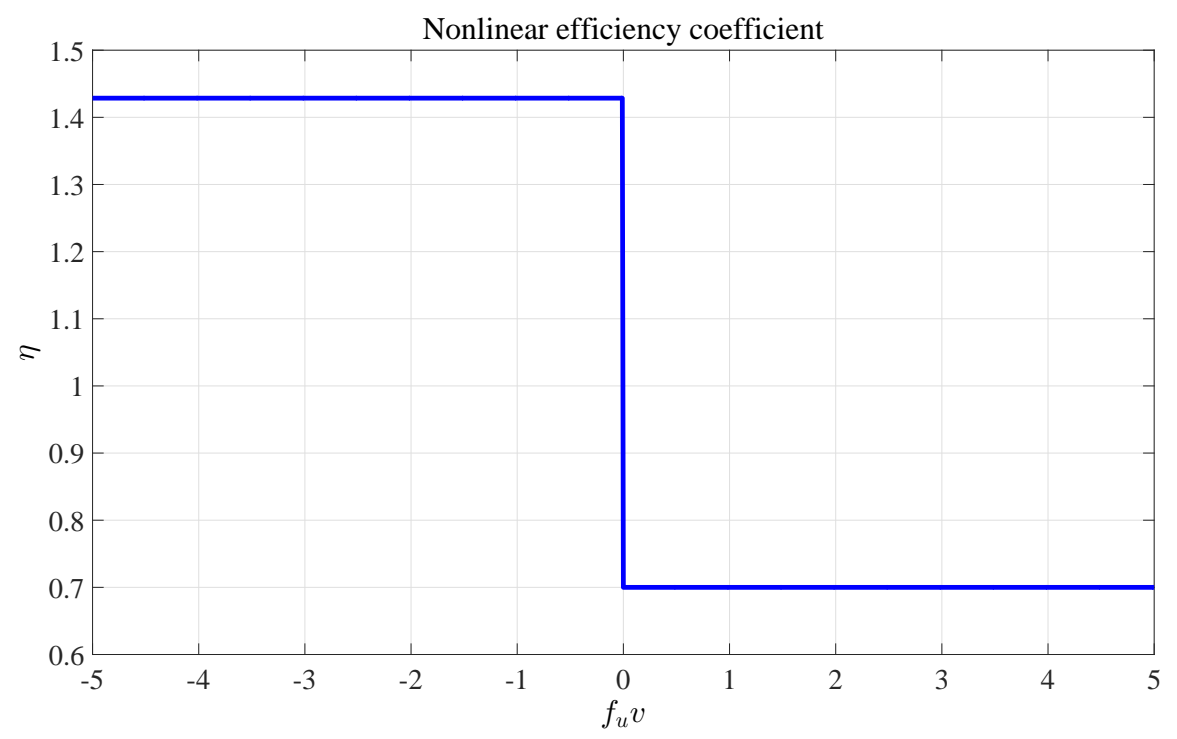

Fig. 2. Nonlinear efficiency coefficient of the PTO system.

The nonlinear efficiency coefficient plays an essential role in optimal control of WECs, and cannot be neglected. Simulation shows that disregarding $\eta$ may result in a negative $P_{a}$, i.e., in wasting grid energy, in the long run, instead of gaining energy from the waves.

\subsection{Intermittent Adaptive PI Control}

The reference control for the WEC system under study is an intermittent adaptive PI algorithm [26]. This method can be decomposed into an off-line and an on-line stage. For the off-line stage, a representative set of sea-states is chosen. Then, for each sea-state of the set, the PI control gains are optimized using a brute-force search. For the on-line stage, the gains are adapted as the sea state changes.

There are two main problems with this intermittent or switching adaptive PI. The first one is that there is no guarantee that the gains are optimal even 
for a fixed sea state. The second one is that the sea state is characterized

by its spectrum, which is in its turn identified by the Fourier transform of the wave elevation. It is well known [8] that a large amount of data with time windows of several minutes needs to be gathered to identify the sea state by its spectrum. Until a new sea state is determined, the PI gains are kept constant. This implies that the PI control law is always suboptimal, since the sea state continuously changes.

\section{Optimal Control in Regular Waves}

\subsection{Average Power Computation}

In a monochromatic sea state, the wave excitation force is given as

$$
f_{e x}(t)=A \sin (\omega t+\phi)
$$

where the phase $\phi$ can be set to be zero without loss of generality. Assuming that the control force is a linear feedback of the heaving velocity, see Fig. 3, its expression in the frequency domain is

$$
f_{u}(j w)=Z_{c}(j w) v(j w)
$$

$Z_{c}$ can be seen as a load impedance, and designed following impedancematching principles [6].

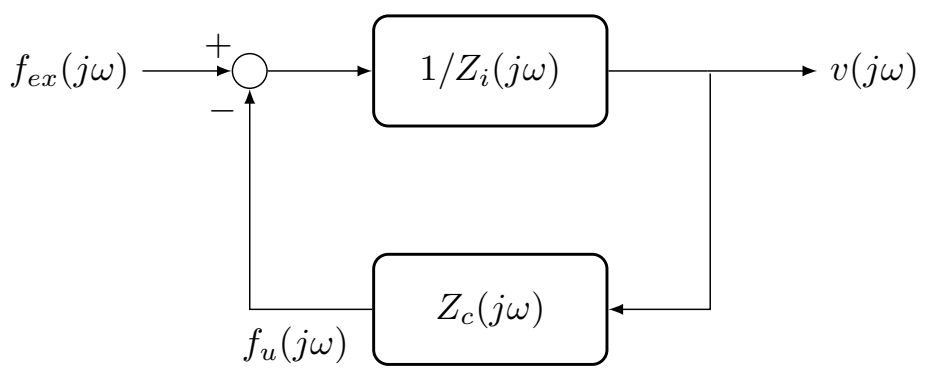

Fig. 3. Block diagram with the WEC $Z_{i}(s)$ and control strategy $Z_{c}(s)$.

Recall that $R_{i}=\operatorname{Re}\left\{Z_{i}(j \omega)\right\}, X_{i}=\operatorname{Im}\left\{Z_{i}(j \omega)\right\}$. Define $R_{c}, X_{c}, \theta$, respectively, as the real part, the imaginary part, and the phase of $Z_{c}(j \omega)$. One has

$$
\left\{\begin{array}{l}
R_{c}=\left|Z_{c}(j \omega)\right| \cos (\theta), \\
X_{c}=\left|Z_{c}(j \omega)\right| \sin (\theta), \\
\theta=\arctan \left(\frac{X_{c}}{R_{c}}\right)
\end{array}\right.
$$


where $\left|Z_{c}(j \omega)\right|$ is the amplitude of $Z_{c}(j w)$. The following theorem holds

Theorem 1: For the given wave excitation force (8) and the control input (9), the average power $P_{a}$ is computed as

$$
P_{a}=\frac{A^{2} R_{c}}{2\left(\left(X_{c}+X_{i}\right)^{2}+\left(R_{c}+R_{i}\right)^{2}\right)}\left(n_{p}-\frac{\eta_{n}-\eta_{p}}{\pi}\left(\frac{X_{c}}{R_{c}}-\arctan \left(\frac{X_{c}}{R_{c}}\right)\right)\right)
$$

Proof: Using (9), the WEC velocity is given as

$$
v(j w)=\frac{1}{Z_{i}(j w)+Z_{c}(j w)} f_{e x}(j w)=W(j w) f_{e x}(j w)
$$

where

$$
W(j w)=\frac{1}{Z_{i}(j w)+Z_{c}(j w)}
$$

is the transfer function of the closed loop system with the wave excitation force as input and the velocity as output. Using (8), and as (12) is a linear system, it follows that $v(t)$ is a sinusoid with the same frequency $\omega$ as $f_{e x}(t)$, but with different amplitude and phase

$$
v(t)=|W(j \omega)| A \sin (\omega t+\angle W(j \omega))
$$

where $|W(j \omega)|$ and $\angle W(j \omega)$ are, respectively, the amplitude and the phase of $W(j w)$.

Analogously, using (9), (13), one obtains

$$
f_{u}(t)=\left|Z_{c}(j \omega)\right||W(j \omega)| A \sin (\omega t+\angle W(j \omega)+\theta)
$$

Combining (13), (14), the instantaneous power is given as

$$
\begin{aligned}
P(t) & =f_{u}(t) v(t) \\
& =\left|Z_{c}(j \omega)\right||W(j \omega)|^{2} A^{2} \sin (\omega t+\angle W(j \omega)) \sin (\omega t+\angle W(j \omega)+\theta)
\end{aligned}
$$

and the average power as

$$
P_{a}=\frac{1}{T} \int_{0}^{T} \eta P(t) d t
$$

For the average power computation, it is clear that only the relative phase shift of two sinusoids is required. Hence $\angle W(j \omega)$ can be omitted. As a result

$$
P_{a}=\frac{1}{T} \int_{0}^{T} \eta P_{m}(t) d t
$$

where

$$
P_{m}(t)=\left|Z_{c}(j \omega)\right||W(j \omega)|^{2} A^{2} \sin (\omega t) \sin (\omega t+\theta)
$$


It suffices to compute $P_{a}$ for one half-period $T=\frac{2 \pi}{\omega}$ since the wave excitation force is periodic. Using (7), (17), it is clear that $P_{a}$ depends on the sign of

$P_{m}(t)$. Note that this sign depends only on the product $\sin (\omega t) \sin (\omega t+\theta)$. Note also that $\sin (\omega t) \geq 0$ on the interval $0 \leq t \leq \frac{\pi}{\omega}$. Hence $P_{m}(t)$ and $\sin (\omega t+\theta)$ have the same sign on the considered interval.

There are two cases, as a function of $\theta$,

(1) If $0 \leq \theta \leq \pi$, then

$$
\left\{\begin{array}{l}
\sin (\omega t+\theta) \geq 0, \text { if } 0 \leq t \leq \frac{\pi-\theta}{\omega} \\
\sin (\omega t+\theta) \leq 0, \text { if } \frac{\pi-\theta}{\omega} \leq t \leq \frac{\pi}{\omega}
\end{array}\right.
$$

And hence,

$$
\left\{\begin{array}{l}
P_{m}(t) \geq 0, \text { if } 0 \leq t \leq \frac{\pi-\theta}{\omega} \\
P_{m}(t) \leq 0, \text { if } \frac{\pi-\theta}{\omega} \leq t \leq \frac{\pi}{\omega}
\end{array}\right.
$$

(2) If $\pi \leq \theta \leq 2 \pi$, then

$$
\left\{\begin{array}{l}
\sin (\omega t+\theta) \leq 0, \text { if } 0 \leq t \leq \frac{-\theta+2 \pi}{\omega} \\
\sin (\omega t+\theta) \geq 0, \text { if } \frac{-\theta+2 \pi}{\omega} \leq t \leq \frac{\pi}{\omega}
\end{array}\right.
$$

And hence,

$$
\left\{\begin{array}{l}
P_{m}(t) \leq 0, \text { if } 0 \leq t \leq \frac{-\theta+2 \pi}{\omega} \\
P_{m}(t) \leq 0, \text { if } \frac{-\theta+2 \pi}{\omega} \leq t \leq \frac{\pi}{\omega}
\end{array}\right.
$$

We will show the proof only for the case 1 , since it is the same for the case 2 . One has

$$
P_{a}=\frac{1}{T}\left(\int_{0}^{\frac{\pi-\theta}{\omega}} \eta_{p} P_{m}(t) d t+\int_{\frac{\pi-\theta}{\omega}}^{\frac{\pi}{\omega}} \eta_{n} P_{m}(t) d t\right)
$$

Using (18), (23) becomes

$$
P_{a}=\frac{\omega}{\pi}\left|Z_{c}(j \omega)\right||W(j \omega)|^{2} A^{2} S(\omega)
$$

where

$$
S(\omega)=\eta_{p} \int_{0}^{\frac{\pi-\theta}{\omega}} \sin (\omega t) \sin (\omega t+\theta) d t+\eta_{n} \int_{\frac{\pi-\theta}{\omega}}^{\frac{\pi}{\omega}} \sin (\omega t) \sin (\omega t+\theta) d t
$$

Using the following product-to-sum trigonometric identity

$$
\sin (\alpha) \sin (\beta)=\frac{1}{2}(\cos (\alpha-\beta)-\cos (\alpha+\beta))
$$


equation (25) is rewritten as

$$
S(\omega)=\frac{\eta_{p}}{2} \int_{0}^{\frac{\pi-\theta}{\omega}}(\cos (\theta)-\cos (2 \omega t+\theta)) d t+\frac{\eta_{n}}{2} \int_{\frac{\pi-\theta}{\omega}}^{\frac{\pi}{\omega}}(\cos (\theta)-\cos (2 \omega t+\theta)) d t
$$

One has

$$
\begin{aligned}
S(\omega) & =\left.\frac{\eta_{p}}{2}\left(\cos (\theta) t-\frac{\sin (2 \omega t+\theta)}{2 \omega}\right)\right|_{t=0} ^{t=\frac{\pi-\theta}{\omega}}+\left.\frac{\eta_{n}}{2}\left(\cos (\theta) t-\frac{\sin (2 \omega t+\theta)}{2 \omega}\right)\right|_{t=\frac{\pi-\theta}{\omega}} ^{t=\frac{\pi}{\omega}} \\
& =\frac{\eta_{p}}{2}\left(\cos (\theta) \frac{\pi-\theta}{\omega}+\frac{\sin (\theta)}{\omega}\right)+\frac{\eta_{n}}{2}\left(\cos (\theta) \frac{\theta}{\omega}-\frac{\sin (\theta)}{\omega}\right) \\
& =\frac{1}{2 \omega}\left(\pi \eta_{p} \cos (\theta)+\left(\eta_{n}-\eta_{p}\right) \theta \cos (\theta)-\left(\eta_{n}-\eta_{p}\right) \sin (\theta)\right)
\end{aligned}
$$

Together with (24), one obtains

$$
\begin{aligned}
P_{a} & =\frac{1}{2 \pi}\left|Z_{c}(j \omega)\right||W(j \omega)|^{2} A^{2}\left(\pi \eta_{p} \cos (\theta)+\left(\eta_{n}-\eta_{p}\right) \theta \cos (\theta)-\left(\eta_{n}-\eta_{p}\right) \sin (\theta)\right) \\
& =\frac{|W(j \omega)|^{2} A^{2}}{2}\left(\eta_{p}\left|Z_{c}(j \omega)\right| \cos (\theta)+\frac{\eta_{n}-\eta_{p}}{\pi}\left(\theta\left|Z_{c}(j \omega)\right| \cos (\theta)-\left|Z_{c}(j \omega)\right| \sin (\theta)\right)\right)
\end{aligned}
$$

Note that

$$
|W(j \omega)|^{2}=\frac{1}{\left(X_{c}+X_{i}\right)^{2}+\left(R_{c}+R_{i}\right)^{2}}
$$

Substituting (10), (28) into (27), one obtains

$$
P_{a}=\frac{A^{2} R_{c}}{2\left(\left(X_{c}+X_{i}\right)^{2}+\left(R_{c}+R_{i}\right)^{2}\right)}\left(n_{p}-\frac{\eta_{n}-\eta_{p}}{\pi}\left(\frac{X_{c}}{R_{c}}-\arctan \left(\frac{X_{c}}{R_{c}}\right)\right)\right)
$$

The proof is complete.

Remark: Consider the limiting case of the ideal PTO, i.e., $\eta_{p}=\eta_{n}=1$. Using Theorem 1, the following average power is obtained

$$
P_{a}=\frac{A^{2} R_{c}}{2\left(\left(X_{c}+X_{i}\right)^{2}+\left(R_{c}+R_{i}\right)^{2}\right)}
$$

Equation (30) is the same as the one in [6]. Hence the classical result [6] is a particular case of Theorem 1, where the nonlinear efficiency coefficient is not taken into account.

\subsection{Optimal Frequency Response}

Using (11), our next step is to compute the optimal $R_{c}, X_{c}$ to maximize $P_{a}$. We require that $R_{c} \geq 0$, since $R_{c}$ is used to convert the wave energy into useful mechanical or electrical energy. The factor $\frac{A^{2}}{2}$ has no influence on the optimal solution, it can be omitted in the optimization problem. Thus, the problem to 
be solved is

$$
\max _{R_{c}, X_{c}}\left\{f\left(R_{c}, X_{c}\right)\right\}
$$

where

$$
\left\{\begin{array}{l}
f\left(R_{c}, X_{c}\right)=g\left(R_{c}, X_{c}\right) h\left(R_{c}, X_{c}\right) \\
g\left(R_{c}, X_{c}\right)=\frac{R_{c}}{\left(X_{c}+X_{i}\right)^{2}+\left(R_{c}+R_{i}\right)^{2}} \\
h\left(R_{c}, X_{c}\right)=n_{p}-\frac{\eta_{n}-\eta_{p}}{\pi}\left(\frac{X_{c}}{R_{c}}-\arctan \left(\frac{X_{c}}{R_{c}}\right)\right)
\end{array}\right.
$$

It is impossible to obtain an analytical form for the optimal solution to (31), since it is a nonlinear optimization problem.

Remark: Note that the optimal solution $\left\{R_{c}, X_{c}\right\}$ does not depend on $A$ due to the efficiency model (7). However, the approach is readily extended to the case when $\eta_{n}, \eta_{p}$ are functions of $A$. As consequence, the optimal solution $\left\{R_{c}, X_{c}\right\}$ also depends on $A$.

We require that $R_{c}, X_{c}$ are such that $h\left(R_{c}, X_{c}\right) \geq 0$, since the harvested average power $P_{a}$ should be nonnegative. Define $\mu=\frac{X_{c}}{R_{c}}$. One has $h\left(R_{c}, X_{c}\right)=$ $h(\mu)$, and

$$
\frac{d h(\mu)}{d \mu}=-\frac{\eta_{n}-\eta_{p}}{\pi} \frac{\mu^{2}}{1+\mu^{2}}<0
$$

It follows that $h(\mu)$ is a monotonically decreasing function, since $\eta_{n} \geq 1 \geq \eta_{p}$. Therefore the equation,

$$
\eta_{p}-\frac{\eta_{n}-\eta_{p}}{\pi}(\mu-\arctan (\mu))=0
$$

has a unique solution $\mu^{*}$. Consequently, the line

$$
X_{c}=\mu^{*} R_{c}
$$

separates the plane $\left(R_{c}, X_{c}\right)$ into two parts

- $h\left(R_{c}, X_{c}\right) \geq 0$ for all $R_{c}, X_{c}$ such that $X_{c} \leq \mu^{*} R_{c}$

- $h\left(R_{c}, X_{c}\right) \geq 0$ for all $R_{c}, X_{c}$ such that $X_{c} \geq \mu^{*} R_{c}$

The functions $g\left(R_{c}, X_{c}\right)$ and $h\left(R_{c}, X_{c}\right)$ are positive and concave for all $R_{c}, X_{c}$ such that $X_{c} \leq \mu^{*} R_{c}$. In addition $h\left(R_{c}, X_{c}\right)$ is monotonic non-increasing. Hence $f\left(R_{c}, X_{c}\right)$ is a concave function [3]. It follows that the solution to (31) is unique and can be found by any constrained optimization method.

Figure 4(a) presents a numerical example of the average power $P_{a}$ as a function of $R_{c}, X_{c}$ for a given and fixed $\omega$, Figure 4(b) shows a contour plot of $P_{a}$ in the $R_{c}-X_{c}$ plane. The efficiency coefficients are $\eta_{p}=0.7, \eta_{n}=1.43$. Using the Newton-Raphson method to solve (34), one obtains $\mu^{*}=4.364$. The WEC system parameters are given in Section 5. 


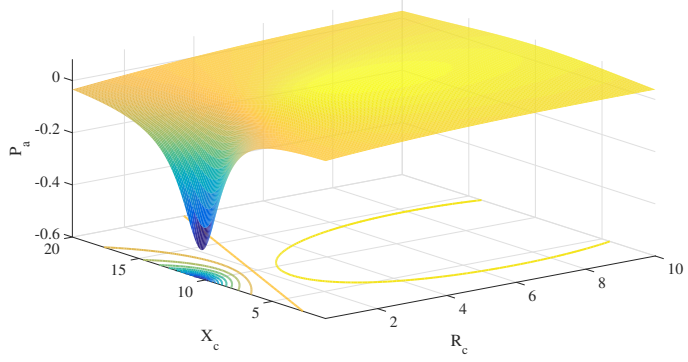

(a) $P_{a}$

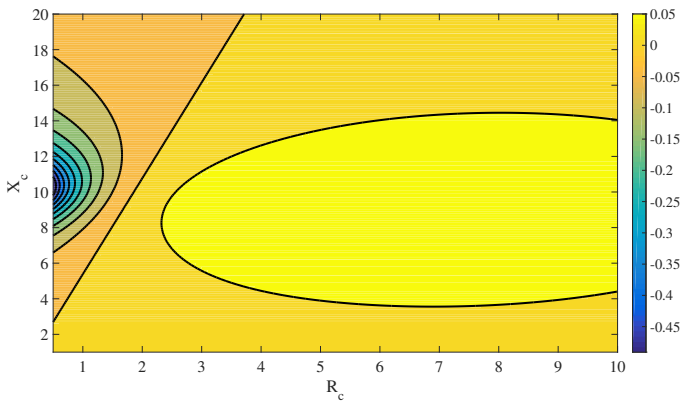

(b) Contour plot $P_{a}$

Fig. 4. (a) Average power $P_{a}$ as a function of $R_{c}$ and $X_{c}$. (b) Contour plot $P_{a}$ in the $R_{c}-X_{c}$ plane

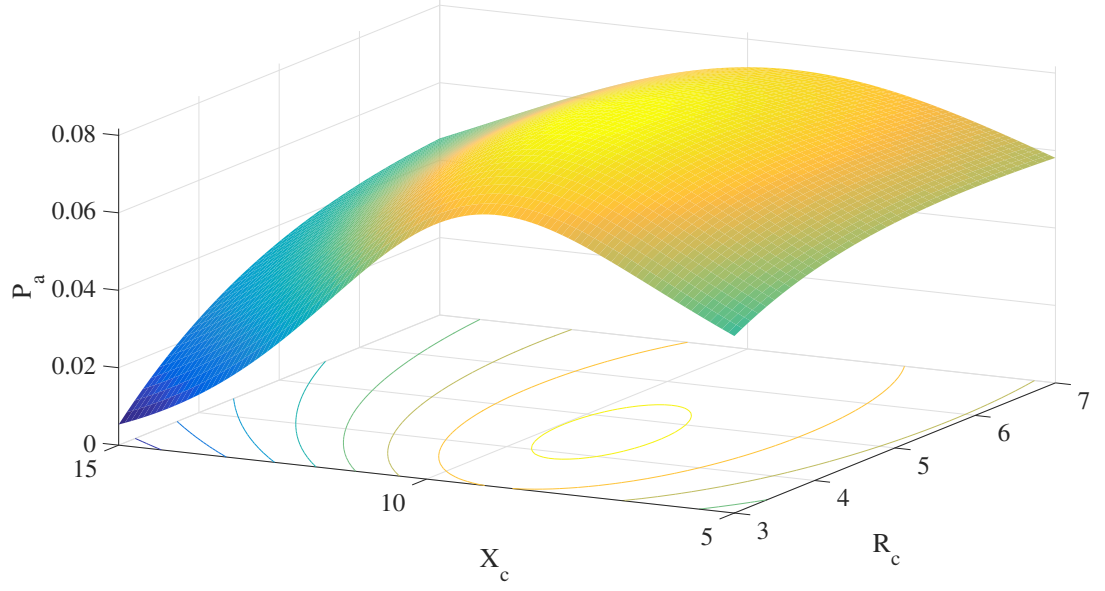

Fig. 5. Average power $P_{a}$ as a function of $R_{c}$ and $X_{c}$ with $X_{c} \leq \mu^{*} R_{c}$.

Figure 5 shows the average power $P_{a}$ in the region where $X_{c} \leq \mu^{*} R_{c}$.

Figure 6(a) shows the optimal $R_{c}^{*}$ (solid blue) and $R_{i}$ (dashed red) as a function of $\omega$. The optimal $X_{c}^{*}$ (solid blue) and $-X_{i}$ (dashed red) are presented in Figure $6(\mathrm{~b})$. It is worth noticing from Figure $6(\mathrm{~b})$ that $X_{c}^{*}$ is very close but not equal to $-X_{i}$. Hence the phase response $\angle W(s)$ of the transfer function (36) of the WEC closed loop system

$$
v(j w)=\frac{1}{Z_{i}(j w)+Z_{c}(j w)} f_{e x}(j w)=W(j w) f_{e x}(j w)
$$

is generally not equal to zero. It follows that, in the presence of the nonlinear efficiency coefficient, the optimal velocity is not in phase with the wave excitation force. The result of Falnes [6], where it is stated that the optimal velocity is in phase with the wave excitation force, can be considered only as an ideal case, where the PTO system is perfect.

Remark: For the given WEC system, it is interesting to observe that $X_{c}$ is monotonically decreasing for the considered frequency interval $1 \mathrm{rad} / \mathrm{s} \leq$ 


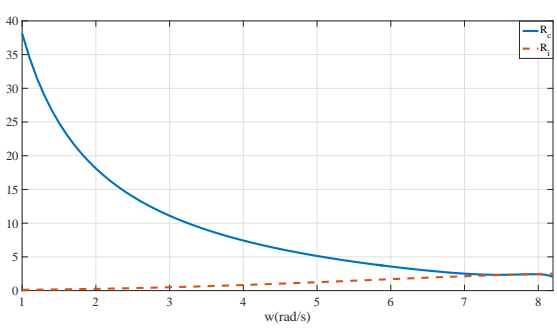

(a) $R_{c}^{*}$

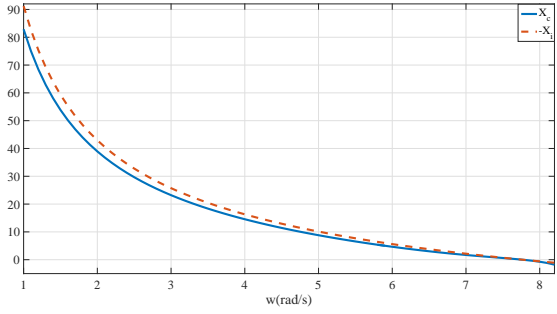

(b) $X_{c}^{*}$

Fig. 6. (a) Optimal $R_{c}^{*}$ (solid blue) and $R_{i}$ (dashed red) as a function of $\omega$. (b) Optimal $X_{c}^{*}$ (solid blue) and $-X_{i}$ (dashed red) as a function of $\omega$.

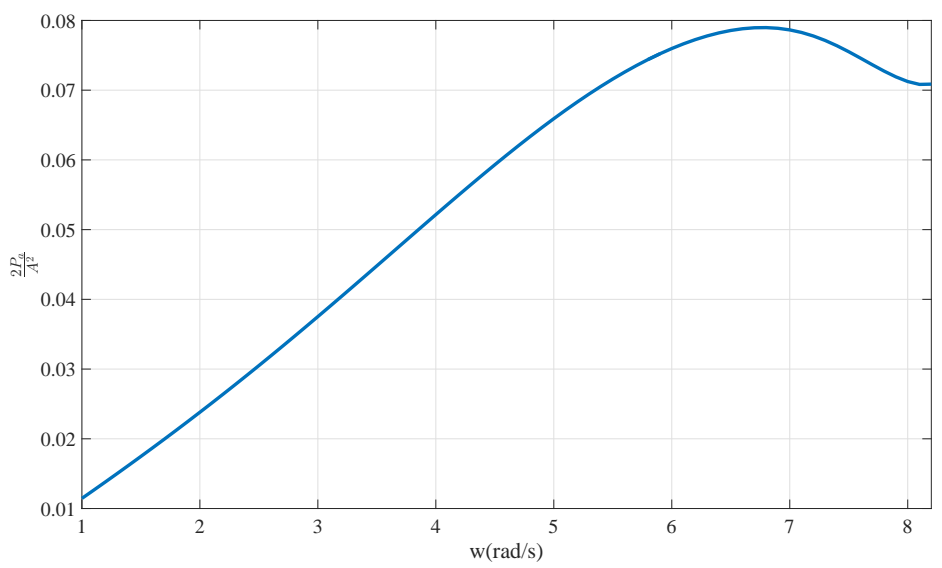

Fig. 7. $\frac{2 P_{a}}{A^{2}}$ as a function of $\omega$.

$175 \omega \leq 8 \mathrm{rad} / \mathrm{s}$. This is explained by the facts that $X_{c}$ is used to change the WEC resonance frequency, and that the resonance frequency of the system is $w_{r}=7.82 \mathrm{rad} / \mathrm{s}$. As a consequence, a relatively large amount of reactive power is required for low frequency waves. However, the overall harvested power should remain non-negative, see Fig. 7 , since $f_{u}=0$ is always a feasible solution.

Remark: If the PTO system is constrained to be purely resistive, then $X_{c}=0$. In this case the optimization problem (31) becomes

$$
\max _{R_{c}}\left\{\frac{n_{p} R_{c}}{X_{i}^{2}+\left(R_{c}+R_{i}\right)^{2}}\right\}
$$

There exists an analytical solution to (37)

$$
R_{c}^{*}=\sqrt{X_{i}^{2}+R_{i}^{2}}
$$

Note that the efficiency coefficient does not play any role in the optimal $R_{c}^{*}$ in the resistive PTO system case. This is explained by the fact that there is no PTO input reactive power, so the energy harvested from wave is always 
positive.

\section{Adaptive PI Control in Irregular Waves}

The algorithm described in Section 3 allows to compute, for a specific WEC and for a given regular wave excitation force, the optimal frequency response $R_{c}^{*}, X_{c}^{*}$ with $R_{c}^{*}=\operatorname{Re}\left\{Z_{c}(j w)\right\}$ and $X_{c}^{*}=\operatorname{Im}\left\{Z_{c}(j w)\right\}$. A control structure needs to be assigned for $Z_{c}$ to employ $R_{c}^{*}, X_{c}^{*}$. In this work, the following proportional-integral (PI) law is chosen

$$
Z_{c}(j w)=K_{p}+\frac{K_{i}}{j w}
$$

It follows that

$$
\left\{\begin{array}{l}
K_{p}=R_{c}^{*}, \\
K_{i}=-\omega X_{c}^{*}
\end{array}\right.
$$

Using (39), the control action is computed in the time domain as

$$
f_{u}(t)=K_{p} v(t)+K_{i} \int_{0}^{t} v(\tau) d \tau
$$

Remark: The "PI" control law is used in this work due to its simplicity. However the other structures, such as "PID" or a high-order controller, can also be used. The main advantage of the "PID" or high-order control law is that they offer additional degrees of freedom to harvest more wave energy.

In the monochromatic sea state (8), the optimal control action is computed as (41), where the PI coefficients are given in (40). Unfortunately, real sea states are polychromatic, i.e., $f_{e x}(t)$ is not a pure sinusoid. In addition, $f_{e x}(t)$ cannot be measured directly when the WEC system is running.

The first problem is addressed by modeling the wave excitation force as a time varying sinusoidal signal, i.e.,

$$
f_{e x}(t)=A(t) \sin (\omega(t) t+\phi(t))
$$

where the parameters $A(t), \omega(t)$ and $\phi(t)$ are estimated in real time. Since $A(t), \omega(t)$ and $\phi(t)$ enter non-linearly in the equation (42), this is a nonlinear estimation problem.

In [9], an extended Kalman filter (EKF) approach is proposed to estimate $A(t), \omega(t), \phi(t)$. However, it must be recalled that the EKF provides a solution to a nonlinear estimation problem via local linearizations of the underlying 
model. Thus, if the variation of the wave excitation force is large and/or the sampling time intervals are not sufficiently small, the linearization may yield highly unstable filters, potentially leading to divergence phenomena [15], [16]. A more robust solution consists in estimating $A(t), \omega(t), \phi(t)$ using an unscented Kalman filter (UKF) is proposed in [24]. The UKF is a powerful nonlinear estimation technique and has been shown to be a superior alternative to the EKF in several applications including parameter estimation for time series modeling [11], neural network training [12], and state estimation for road vehicle navigation [13]. An interesting feature of UKF is that no linearization of the nonlinear model is required.

As to wave excitation force estimation, a few methods have been proposed recently and tested in real-time, in a model predictive control (MPC) framework, see for instance [18], [10], [20], [21]. The method presented and assessed in [21], based on a linear Kalman filter and a random walk model for the variation of the wave excitation force, has the main advantages of using only standard measurements for reactive control, i.e., position, velocity, PTO force and of yielding quite accurate estimates over a large range of sea states.

The adaptive PI control strategy is summarized as follows.

Offline stage: For each frequency $\omega$

(1) Compute $R_{i}, X_{i}$ of $Z_{i}(j w)$ as $R_{i}=\operatorname{Re}\left(Z_{i}(j w)\right)$ and $X_{i}=\operatorname{Im}\left(Z_{i}(j w)\right)$.

(2) Compute the optimal $R_{c}^{*}, X_{c}^{*}$ by solving the optimization problem (31) with constraints $R_{c} \geq 0$, and $X_{c} \leq \mu^{*} R_{c}$.

(3) Compute the optimal PI gains $K_{p}, K_{i}$ using (40).

Online stage: At each sampling time $t$

(1) Estimate $\hat{f}_{e x}(t)$ using the measured WEC position, and the measured WEC velocity.

(2) Estimate $\hat{\omega}$ using the UKF approach.

(3) Compute the coefficients $K_{p}, K_{i}$ as (41).

(4) Compute the control action as

$$
f_{u}(t)=K_{p} v(t)+K_{i} p(t)
$$

The adaptive PI algorithm is schematically presented in Figure 8. It is worth noticing that only the measurements of the WEC position and the WEC speed are required. No information about $f_{e x}(t)$ is needed.

Remark: For simplicity, the case when $\eta_{n}, \eta_{p}$ are independent of the sea state is considered. However, the proposed approach is straightforwardly extended to the case when $\eta_{n}, \eta_{p}$ depend on the sea state. The unscented Kalman filter 


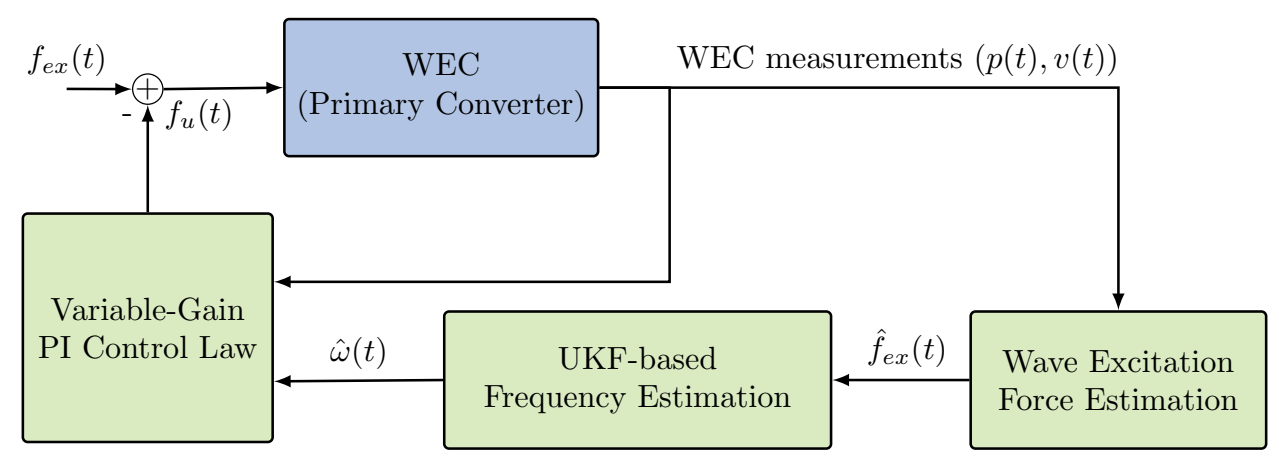

Fig. 8. Adaptive PI control approach.

can estimate not only the dominant wave frequency, but also the dominant wave amplitude, which are the two characterizing parameters for a given sea state. By solving the optimization problem (31) offline, one can obtain the optimal parameters $\left\{R_{c}, X_{c}\right\}$, or equivalently the $\left\{K_{i}, K_{p}\right\}$ gains. For the online implementation, this yields a two-dimensional look-up table, where the dominant amplitude and the dominant frequency are parameters, instead of a one-dimensional look-up table as in the paper.

\section{Experimental Results}

\subsection{Experimental Setup}

A laboratory prototype of a point absorber WEC [28] on a 1:20 scale with respect to the well-known Wavestar machine installed near Hanstholm in Denmark from 2009 to 2013, was used to validate the adaptive PI control algorithm described in Section 4. As it can be seen in Fig. 9, the prototype consists of a float attached to an arm, which in turn is attached to a PTO emulator. The laboratory model is equipped with a laser and an acceleration sensor to measure, respectively, the position and the acceleration. The velocity of the float can then be estimated from these measurements using a linear Kalman filter. A linear electrical generator is chosen to emulate the PTO system. It applies a force to the arm, which can be measured by a load cell, see Fig. 9 and used to compute the equivalent moment applied to the float. The WEC controller sends a force reference to a low-level force servo-controller that operates the generator.

Remark: Since the motion of the considered WEC system is angular, Newton's second law for rotation is used to obtain a mathematical model. The quantity that fully describes the system in this case is the angular deviation from the equilibrium point. The changes that take place are that the equivalent mass changes to an equivalent moment inertia, and the acting forces 
become acting moments.

The experimental work was carried out in a wave basin at Aalborg University, Denmark, see Fig. 10. It consists of four parts: the wave generation (a), the wave elevation (b), the point absorber model (c), and the wave termination (d). The basin has a length of $15 \mathrm{~m}$, a width of $8 \mathrm{~m}$ and a maximum water depth of $0.7 \mathrm{~m}$. The wave paddles are driven by a total of 15 hydraulic pistons moving in the horizontal direction. The waves were generated by the wave maker based on a Pierson-Moskowitz spectrum. Three distinguished features of the system were used thoroughly for testing. First the paddle movement can be stored during a test run and then can be reproduced in further test runs. Secondly, a trigger signal is sent by the wave generator, allowing exact alignment of the tests. Thirdly, the torque sensor of the PTO can be used to measure the wave excitation moment, for the case, when

- The control is not active, i.e. the PTO system does not introduce any torque to the system.

- At the same time the float is manually blocked at equilibrium position.

These three features allow to compare the measured wave excitation moment from the first test to estimated wave excitation moment from the other tests, where the float is moving and the PTO is turned on.
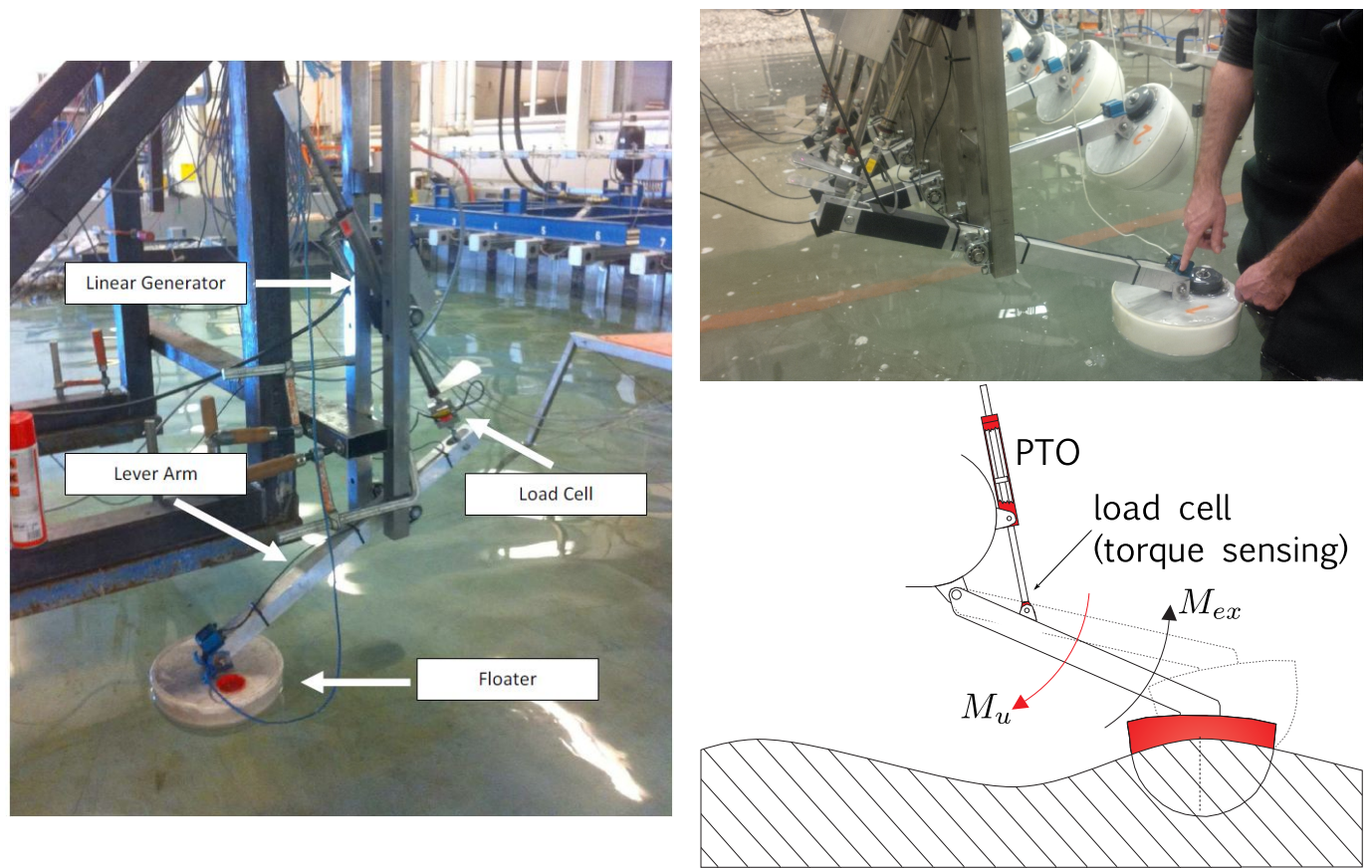

Fig. 9. Experimental set-up. 


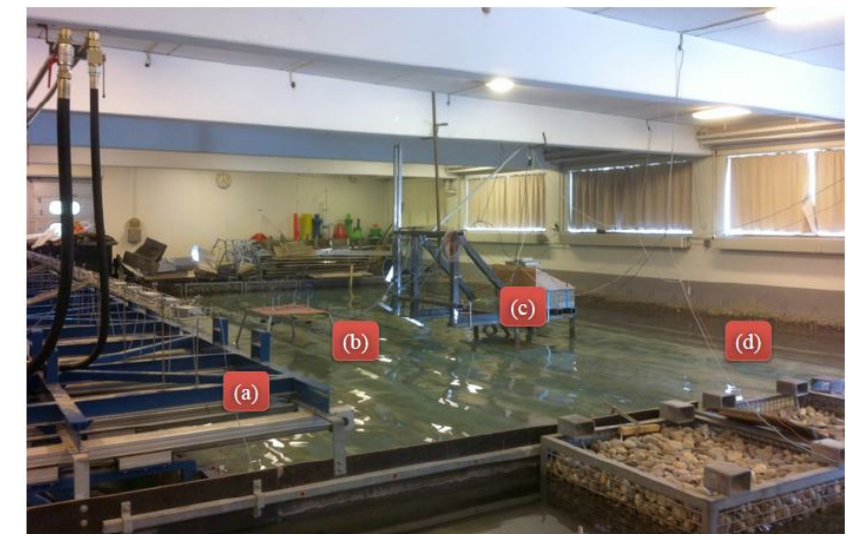

Fig. 10. Experimental setup at AAU. The waves are generated by the wave generation system (a) and the wave elevation is measured by a series of wave gauges (b). The waves then reach the WEC model, where the wave energy absorption takes place (c). Finally, they reach the beach where the termination takes place by a scattering process $(d)$.

The WEC transfer function is given as [13], [26]

$$
Z_{i}(s)=\frac{s^{6}+a_{5} s^{5}+a_{4} s^{4}+a_{3} s^{3}+a_{2} s^{2}+a_{1} s}{b_{7} s^{7}+b_{6} s^{6}+b_{5} s^{5}+b_{4} s^{4}+b_{3} s^{3}+b_{2} s^{2}+b_{1} s+b_{0}}
$$

whose coefficients are given in Table 1.

Table 1

\begin{tabular}{|l|l|}
\hline Numerator & Denominator \\
\hline & $b_{7}=1.44$ \\
& $b_{6}=300.4$ \\
$a_{5}=208.6$ & $b_{5}=1.237 \times 10^{5}$ \\
$a_{4}=8.583 \times 10^{4}$ & $b_{4}=1.284 \times 10^{7}$ \\
$a_{3}=8.899 \times 10^{6}$ & $b_{3}=1.652 \times 10^{8}$ \\
$a_{2}=1.074 \times 10^{8}$ & $b_{2}=2.106 \times 10^{9}$ \\
$a_{1}=7.031 \times 10^{8}$ & $b_{1}=9.988 \times 10^{9}$ \\
& $b_{0}=6.539 \times 10^{10}$ \\
\hline
\end{tabular}

WEC model coefficients

Figure 11(a) presents the Bode plot of the considered WEC system. The resonance frequency is $w_{r}=7.84 \mathrm{rad} / \mathrm{s}$. This Bode plot is very typical for a WEC system of the point-absorber type.

The following input constraints

$$
-6.25 \leq f_{u} \leq 6.25
$$




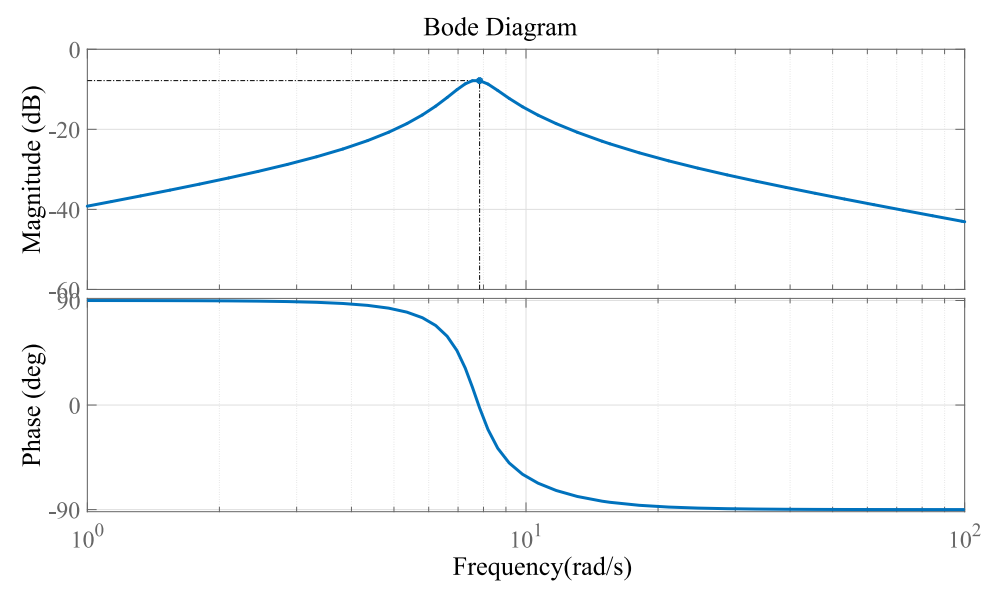

Fig. 11. Bode plot of the considered WEC system

are also considered. Note that for this adaptive PI algorithm such constraints are not yet taken into account in computing the optimal gains.

\subsection{Experimental Results}

In this section, we present experimental results obtained using the control algorithm in Section 4, and a PI control, which is the state of the art control law for the considered WEC system [13], [26]. The PI gains are obtained offline using a griding approach.

The experiments were carried out for two different waves defined using a set of three sea states representative of normal operating conditions for this WEC. Figure 12 shows the spectral power density of the two waves. It is important to point out that wave 1 corresponds to one constant sea state (sea state 1), while wave 23 represents the transition between the two most frequent sea states (sea state 2 and 3), which containing much more energy than sea state 1 (as can be seen from the spectra). Wave 23 is used to validate the proposed control algorithm against the fact that the sea state changes continuously.

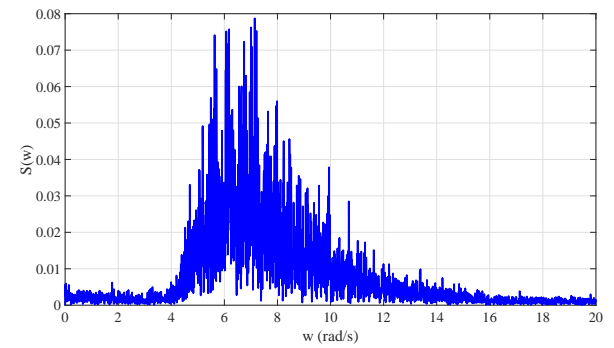

(a) Wave 1

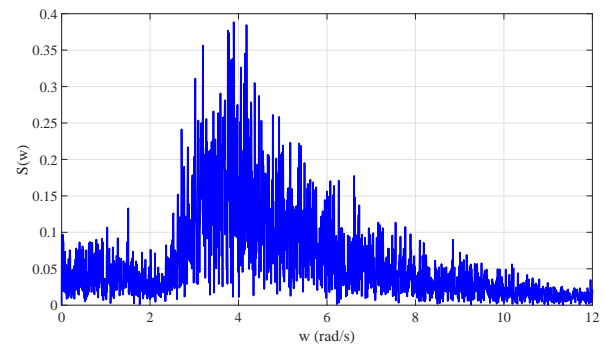

(b) Wave 23

Fig. 12. Wave spectra of sample data set. 
Figure 13 shows the estimated wave frequency $\hat{\omega}$ and wave amplitude $\hat{A}$ as a function of time using the UKF filter for wave 1 . It can be seen that $\hat{\omega}$ is around $6(\mathrm{rad} / \mathrm{s})$ and $7(\mathrm{rad} / \mathrm{s})$ which correspond to the peak of the spectrum of wave 1.

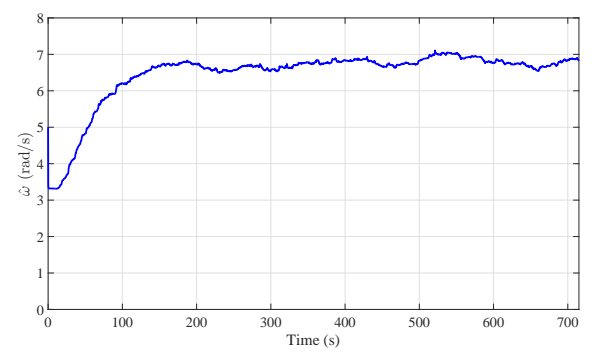

(a) $\hat{\omega}$

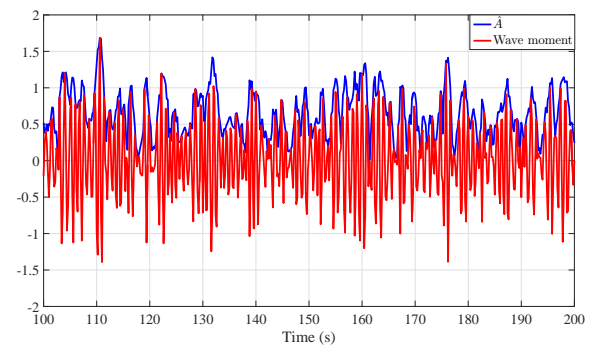

(b) $\hat{A}$

Fig. 13. Time-varying sinusoidal approximation of wave 1 using UKF filter. (a) Estimated wave frequency $\hat{\omega}$. (b) Estimated wave amplitude $\hat{A}$ (solid blue) and wave excitation moment (solid red).

Figure 14(a) shows the float velocity (solid blue) as well as the wave excitation moment (dashed red) as a function of time. Figure 14(b) presents the control input.

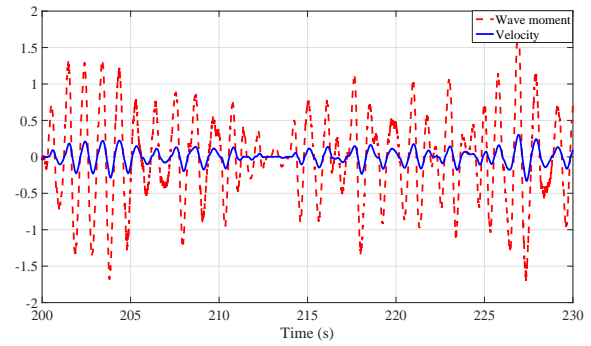

(a) Velocity and wave moment

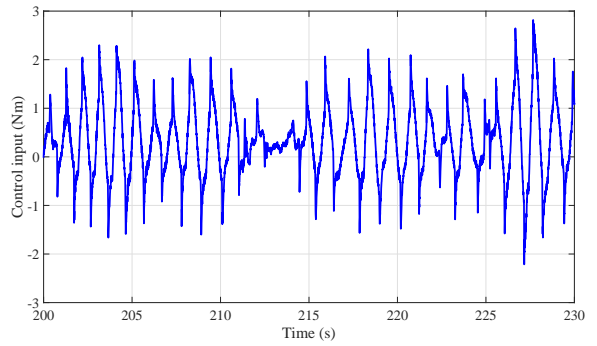

(b) Control input

Fig. 14. Experimental results for wave 1. (a) Float velocity (solid blue) and wave excitation moment (dashed red). (b) Control input.

Figure 15(a) shows the instantaneous powers of the adaptive PI control (solid blue) and of the PI control (solid red) as a function of time. It is worth noticing that the fixed PI control tries to avoid large reactive power excursions. Improvements brought by the adaptive PI strategy are easy to see on Figure 15(b), where the harvested energies of the adaptive PI control (solid blue) and of the PI control (dashed red) are presented. For wave 1, the gain of using the adaptive PI algorithm is $13.86 \%$ with respect to the fixed PI strategy. It should be said that in real-life conditions, the adaptive PI control can be expected to harvest even more energy than the fixed-gain PI control, since it is pre-computed for wave 1 for this experiment. 


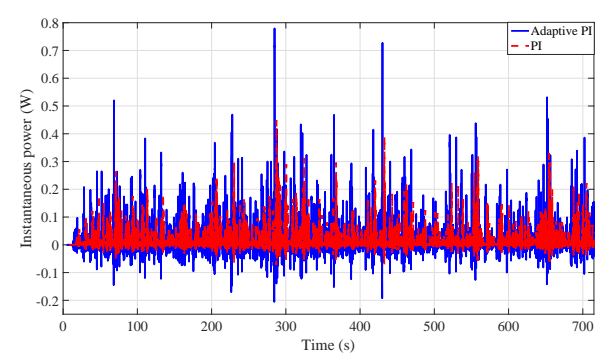

(a) Instantaneous power

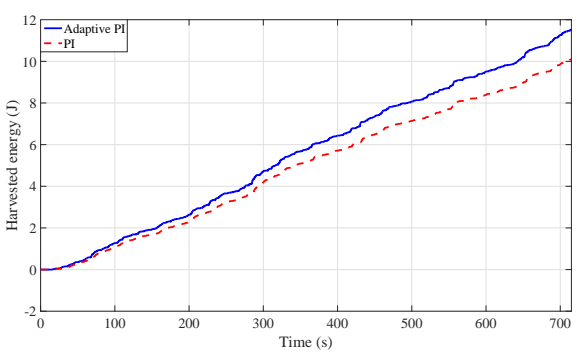

(b) Harvested energy

Fig. 15. Experimental results for wave 1. (a) Instantaneous power for adaptive PI control (solid blue) and for PI control (solid red). (b) Harvested energy for for adaptive PI control (solid blue) and for PI control (dashed red).

Now we will demonstrate the performance of the adaptive PI strategy for wave 23 , which represents a time-varying sea state. Figure 16 shows the performance of the UKF filter used to approximate wave 23 as a time-varying sinusoidal signal. It can be observed that the estimated frequency of wave 23 varies in a much larger interval than the estimated frequency of wave 1.

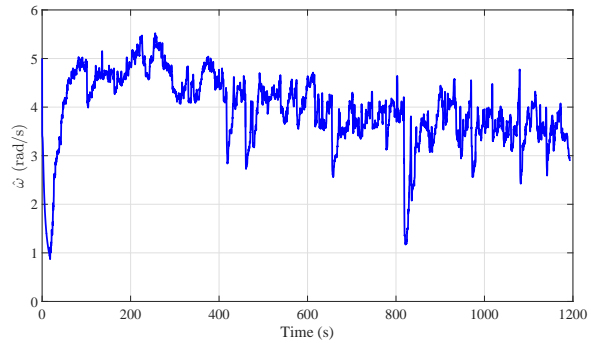

(a) $\hat{\omega}$

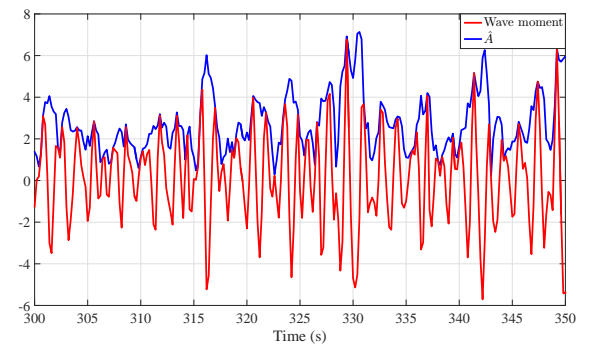

(b) $\hat{A}$

Fig. 16. Time-varying sinusoidal approximation of wave 23 using UKF filter. (a) Estimated wave frequency $\hat{\omega}$. (b) Estimated wave amplitude $\hat{A}$ (solid blue) and wave excitation moment (solid red).

Figure 17(a) presents the float velocity (solid blue) as well as the wave excitation moment (dashed red), while Figure 17(b) shows the control input as a function of time.

Finally, Figure 18(a) shows the obtained instantaneous power of the adaptive PI algorithm (solid blue), and of the fixed PI strategy (solid red). The gains of the fixed PI are optimized for sea state 3, which contains much more energy than sea state 2 . Of course, for such a time-varying sea state, the performance of the reference PI controller could be improved by switching between from the optimal PI gains for sea states 2 to the optimal PI gains for sea state 3, when we are "close" to sea state 3 . Due to the limited time available for the tests, it was not possible to implement a controller reproducing this switching PI behavior. Nevertheless, assuming that that this behavior can be obtained 


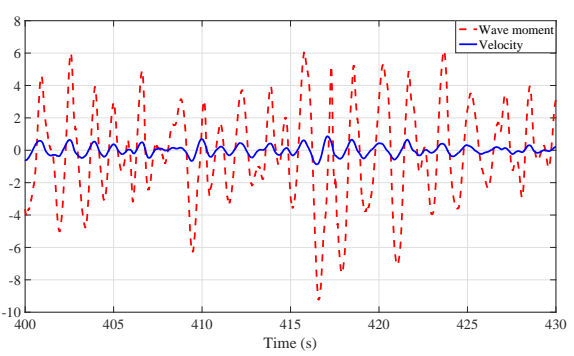

(a) Velocity and wave moment

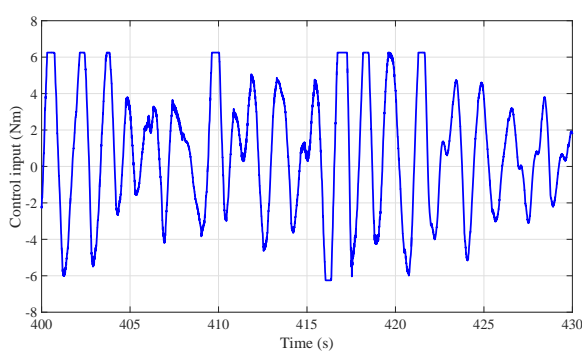

(b) Control input

Fig. 17. Experimental results for wave 23. (a) Float velocity (solid blue) and wave excitation moment (dashed red). (b) Control input.

in real-life conditions, which is questionable since the wave is not known in advance, the benefits of using an adaptive PI instead of a switching PI in the long run should be evident. The adaptive PI can take advantage of each time interval where the switching PI gains are kept fixed, to increase its lead. Figure 18(b) presents the harvested energy of the adaptive PI control (solid blue), and of the fixed-gain PI control (dashed red). Using the adaptive PI control, we harvest $57.14 \%$ more energy than the fixed-gain PI control.

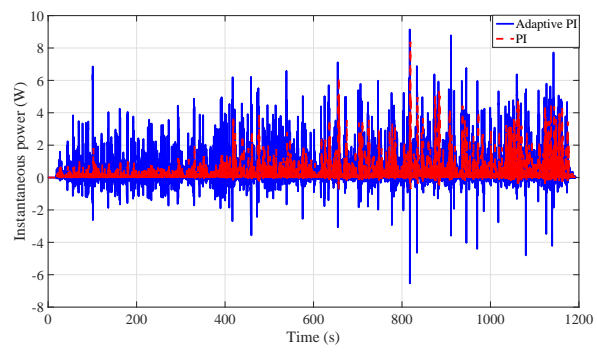

(a) Instantaneous power

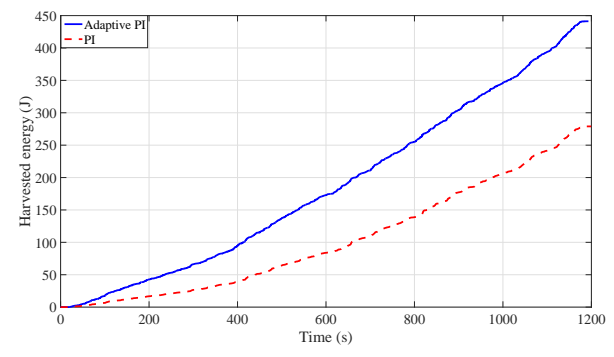

(b) Harvested energy

Fig. 18. Experimental results for wave 23. (a) Instantaneous power for adaptive PI control (solid blue) and for PI control (solid red). (b) Harvested energy for for adaptive PI control (solid blue) and for PI control (dashed red).

\section{Conclusions}

A new approach is proposed to design a continuously adaptive proportionalintegral (PI) velocity-feedback control strategy for wave energy converters. The optimal PI gains are computed based on the solution of a convex optimization problem taking into account non-ideal PTO efficiency. It is shown that the optimal velocity is generally not in phase with the wave excitation force when realistic PTO efficiencies are considered. The gains of the PI controller are continuously adapted on-line, on a wave-to-wave basis, using two robust and accurate methods for real-time estimation of the wave excitation 
force, and of the frequency of the estimated wave excitation force. A case study, based on a lab-scale point-absorber WEC, has been used for validation. Experimental results show that the proposed adaptive control scheme can recover more energy than a fixed-gain PI controller even in a sea state with constant spectral characteristics.

In the future, a more extensive assessment is planned, both in simulation and through experimentation. Though the proposed adaptive PI control system has already been tested in a wave basin on the lab-scale prototype used as a case study, with encouraging results, further experiments are needed to precisely quantify its energy harvesting potential. Finally, further research is needed to take into account WEC motion or actuator constraints, i.e., PTO force limits, stroke limits, which are not dealt with in the current optimization procedure, as well as more complex WEC dynamics.

\section{References}

[1] Aurélien Babarit and Alain H Clément. Optimal latching control of a wave energy device in regular and irregular waves. Applied Ocean Research, 28(2):7791, 2006.

[2] Giorgio Bacelli and John V Ringwood. Numerical optimal control of wave energy converters. IEEE Transactions on Sustainable Energy, 6(2):294-302, 2015 .

[3] Stephen Boyd and Lieven Vandenberghe. Convex optimization. Cambridge university press, 2004.

[4] Julien AM Cretel, Gordon Lightbody, Gareth P Thomas, and Anthony W Lewis. Maximisation of energy capture by a wave-energy point absorber using model predictive control. IFAC Proceedings Volumes, 44(1):3714-3721, 2011.

[5] WE Cummins. The impulse response function and ship motions. Technical report, DTIC Document, 1962.

[6] Johannes Falnes. Ocean waves and oscillating systems: linear interactions including wave-energy extraction. Cambridge university press, 2002.

[7] Johannes Falnes. A review of wave-energy extraction. Marine Structures, 20(4):185-201, 2007.

[8] Thor I Fossen. Handbook of marine craft hydrodynamics and motion control. John Wiley \& Sons, 2011.

[9] Francesco Fusco and John V Ringwood. A simple and effective real-time controller for wave energy converters. Sustainable Energy, IEEE Transactions on, 4(1):21-30, 2013. 
[22] Hoai-Nam Nguyen, Guillaume Sabiron, Paolino Tona, Morten Mejlhede Kramer, and Enrique Vidal Sanchez. Experimental validation of a nonlinear mpc strategy for a wave energy converter prototype. In ASME 2016 35th International Conference on Ocean, Offshore and Arctic Engineering, pages 

2016.

[23] Hoai-Nam Nguyen and Paolino Tona. Continuously adaptive PI control of wave energy converters under irregular sea-state conditions. In 12th European Wave and Tidal Energy Conference 2017, 2017.

[24] Hoai-Nam Nguyen, Paolino Tona, and Guillaume Sabiron. Dominant wave frequency and amplitude estimation for adaptive control of wave energy converters. In OCEANS 2017-Aberdeen, pages 1-6. IEEE, 2017.

[25] SH Salter. The development of the duck concept. In Proc. Wave Energy Conference, pages 17-27, 1978.

[26] Enrique Vidal Sánchez, Rico Hjerm Hansen, and Morten Mejlhede Kramer. Control performance assessment and design of optimal control to harvest ocean energy. IEEE Journal of Oceanic Engineering, 40(1):15-26, 2015.

[27] Trevor Whittaker, David Collier, Matt Folley, Max Osterried, Alan Henry, and Michael Crowley. The development of oyster - a shallow water surging wave energy converter. In Proceedings of the 7th European Wave and Tidal Energy Conference, Porto, Portugal, pages 11-14, 2007.

[28] A Zurkinden, MM Kramer, F Ferri, and JP Kofoed. Numerical modelling and experimental testing of a wave energy converter. Technical report, Tech. report, Department of Civil Engineering, AAU, 2012. 